\title{
Measuring of China's Provincial Carbon Abatement Cost and its Spatial Distribution Pattern
}

\author{
Zhangwen $\mathrm{Li}^{1, *}$, Caijiang Zhang ${ }^{1}$ \\ ${ }^{1}$ South China University of Technology, School of Economics \& Finance, Guangzhou, China
}

\begin{abstract}
Based on the panel data in Chinese provinces from 2000 to 2017, this paper first uses the parameterized quadratic function of the directional distance function to estimate carbon abatement costs of 30 provinces in China, and further studies its long-term evolutionary characteristics. Second, this paper studies the spatial distribution pattern of carbon abatement cost. The results show that the carbon abatement cost has increased as a whole during the study period. Moreover, the spatial distribution of carbon abatement costs in China shows a geographical clustering feature, and the positive spatial agglomeration is significant after 2008.
\end{abstract}

\section{Introduction}

The burning of fossil fuels has produced a great amount of carbon dioxide worldwide since the Industrial Revolution. The contradiction between resource environmental constraints and economic development has become increasingly prominent. Therefore, it is a common responsibility for all countries to take measures to reduce carbon emissions, especially for China. Recently, China government reiterated that China will increase its nationally determined contribution, strive to reach the carbon peak by 2030 and achieve carbon neutrality by 2060. To achieve the carbon emission reduction targets, it is crucial to analyze the long-term evolutionary characteristics of marginal carbon abatement cost (MAC).

The carbon abatement costs have attracted the widespread attention of scholars. For example, Choi et al. (2012) employed the dual model of the slacks-based Data Envelopment Analysis (DEA) model to estimate the abatement costs of $\mathrm{CO}_{2}$ emissions[1]. Duan et al. (2018) calculated the marginal abatement costs of carbon emissions in different ways, found that the carbon abatement cost in all provinces raised from 2005 to 2015[2]. Xue (2021) explored the convergence of MAC during 2001-2015 and pointed that the spatial effect should not be ignored when studying the marginal abatement cost[3]. Actually, rare studies have empirically analyzed the spatial autocorrection of MAC. Thus, to supply for the existing literature, this paper employs a Directional Distance Function (DDF) to estimate the marginal carbon abatement costs of 30 provinces in China and analyze the long-term evolutionary characteristics of MAC. Whereafter, we calculate the Moran's I at the global and local scale to analyze the spatial correction of MAC.

\section{Methodology and data}

\subsection{Measuring the carbon abatement cost}

This paper use DDF to estimate carbon abatement cost. Consider there is a producer who uses inputs $\boldsymbol{x}$ to produce desirable outputs $\boldsymbol{y}$. However, undesirable outputs $\boldsymbol{b}$ are also produced in the meantime. Hence, the production function of the producer shows in formula (1):

$$
P(x)=\{(y, b): x \text { can produce }(y, b)\}
$$

Due to the translation property, we use a quadratic form of the directional distance function expressed as formula (2). We select capital, labor, and energy consumption as input indicators. GDP was determined as the desirable output, and carbon emissions were adopted as the undesirable output.

$$
\begin{aligned}
\vec{D}(x, y, b, g)= & \alpha_{0}+\sum_{n=1}^{3} \alpha_{n} x_{n}+\frac{1}{2} \sum_{n=1}^{3} \sum_{n^{\prime}=1}^{3} \alpha_{n n^{\prime}} x_{n} x_{n^{\prime}} \\
& +\beta_{1} y+\frac{1}{2} \beta_{2} y+\sum_{n=1}^{3} \delta_{n} x_{n} y+\mu y b \\
& +\gamma_{1} b+\frac{1}{2} \gamma_{2} b^{2}+\sum_{n=1}^{3} v_{n} x_{n} b
\end{aligned}
$$

where $\alpha_{0}, \alpha_{n n}, \alpha_{n n^{\prime}}, \beta_{1}, \beta_{2}, \delta_{n}, \mu, \gamma_{1}, \gamma_{2}$ and $v_{n}$ are the parameters to be estimated for $n$ and $n^{\prime}=1,2,3$. $g=(1,-1)$.

$$
\vec{D}_{i}(x, y, b, g)=0
$$

As suggested by Färe et al., the directional distance function can be calculated from the following linear minimization problem[4]:

\footnotetext{
*Corresponding author: lzw1226x@163.com
} 


$$
\begin{aligned}
& \min \sum_{i=1}^{30}\left\{\vec{D}_{i}(x, y, b ; g)\right\}-0 \\
& \text { s.t. } \\
& \text { (1) } \overrightarrow{D_{i}}(x, y, b ; g) \geq 0, i=1, \ldots, 30 \\
& \text { (2) } \partial \overrightarrow{D_{i}}(x, y, b ; g) / \partial y_{i} \leq 0, i=1, \ldots, 30 \\
& \text { (3) } \partial \overrightarrow{D_{i}}(x, y, b ; g) / \partial b_{i} \geq 0, i=1, \ldots, 30 \\
& \text { (4) } \partial \overrightarrow{D_{i}}(x, y, b ; g) / \partial x_{i} \leq 0, i=1, \ldots, 30 \\
& \text { (5) } \alpha_{n^{\prime} n}=\alpha_{n n^{\prime}}, n \neq n^{\prime}, n, n^{\prime}=1,2,3 \\
& \text { (6) } \hat{\beta}_{1}-\hat{\gamma}_{1}=-1, \hat{\beta}_{1}-\hat{\gamma}_{1}=-1, \hat{\delta}_{n}=\hat{v}_{n} \\
& \text { (7) } g=(1,-1)
\end{aligned}
$$

This paper uses Shepard's Lemma, $p$ represents the market price of the desirable output, denote $p=1$, thus, we can derive the carbon abatement cost $q$ as formula (5):

$$
\begin{aligned}
q_{i} & =p\left[\frac{\partial \vec{D}_{i}(x, y, b, g) / \partial b_{i}}{\partial \vec{D}_{i}(x, y, b, g) / \partial y_{i}}\right] \\
& =\frac{\partial \vec{D}_{i}(x, y, b, g) / \partial b_{i}}{\partial \vec{D}_{i}(x, y, b, g) / \partial y_{i}}
\end{aligned}
$$

\subsection{Variable selection}

Input indicators in the directional distance function are capital, labor, and energy consumption. Thus, the capital is represented by fixed asset investment ${ }^{1}$, the labor force is measured by the number of year-end employees, and the energy consumption is represented by the total consumption of fossil fuels. Moreover, the desirable output indicator, GDP, is measured by the real GDP of each province. As for the undesirable output, carbon emission is calculated as follows:

$$
C E=\sum_{m=1}^{17} E C_{m} \times N C V_{m} \times C C_{m} \times O_{m} \times \frac{44}{12}
$$

where $N C V_{m}$ denotes net calorific value of the $m$ th type of fossil fuel, $C C_{m}$ is the carbon content, and $O_{m}$ is oxygenation efficiency of the $j$ th type of fossil fuel. $44 / 12$ represents the ratio of the molecular weight of carbon dioxide to carbon atom.

\subsection{Measuring the dynamic evolution of carbon abatement cost}

This paper employs Kernel density estimation (KDE) to describe the actual data distribution and analyze the dynamic evolution of MAC in China. The KDE can be defined as:

$$
\hat{f}=(1 / n h) \sum_{i=1}^{n} K\left(\left(x-X_{i}\right) / h\right)
$$

Where $\hat{f}$ represents the kernel density value, $h$

\footnotetext{
${ }^{1}$ The capital input variable was calculated by the perpetual
}

represents the bandwidth of $\mathrm{KDE}, K(x)$ denotes the Gaussian kernel function, $x$ represents the estimating site, $X_{i}$ is the number $i$ sample site. $K(x)$ is expressed as follow:

$$
K(x)=(1 / \sqrt{2 \pi}) \cdot \exp \left(-x^{2} / 2\right)
$$

\subsection{Spatial autocorrelation test}

\subsubsection{Global spatial autocorrelation}

To test whether there is a spatial correction in provincial MAC, we employ the global Moran's I index proposed by Cliff and Ord (1981). The specific formula of Moran's I is:

$$
I=\frac{\sum_{i=1}^{30} \sum_{j=1}^{30} w_{i j}\left(Y_{i}-\bar{Y}\right)\left(Y_{j}-\bar{Y}\right)}{S^{2} \sum_{i=1}^{30} \sum_{j=1}^{30} w_{i j}}
$$

where $W_{i j}$ is the spatial weight matrix between region $i$ and region $j ; Y$ is the MAC; $S^{2}$ is the variance of samples. The value of Moran's I is between -1 to 1 , when the value of $I$ is bigger than 0 , it indicates that there is a positive spatial correlation, the larger the value of I, the stronger the positive correlation; otherwise, it indicates that there is a negative spatial correlation; When $\mathrm{I}=0$, it indicates that there is no spatial correlation.

\subsubsection{Local spatial autocorrelation}

Anselin (1995) proposed local indicators of spatial association (LISA), which decomposite global Moran's I into the contribution of each observation [5]. This paper uses the Local Moran's I to explore the statistically significant spatial clusters and dispersion of the provinces' MAC. The calculation of Local Moran's I show below:

$$
I_{i}=\frac{\left(y_{i}-\bar{y}\right)}{S^{2}} \sum_{j=1, j \neq i}^{30} w_{i j}\left(y_{j}-\bar{y}\right)
$$

when the value of $I_{i}$ is positive, there exists a HighHigh (H-H) agglomeration or Low-Low (L-L) agglomeration, while a negative $I_{i}$ indicates a H-L or L$\mathrm{H}$ agglomeration.

\subsection{Data sources}

The data sample of this paper cover 30 Chinese provinces from 2000 to 2017 . The original data are collected from the China Statistical Yearbook (2001-2018) and each province's Statistical Yearbook (2001-2018). The consumption of fossil fuel is derived from the China Energy Statistical Yearbook (2001-2018). We take a moving average approach to solve the problem of few outliers.

inventory method based on the 2000 base period fixed asset investment of each province. 


\section{Result analysis}

\subsection{Time series characteristics of the carbon abatement cost in provinces}

This paper uses MATLAB software to calculate and compare the carbon marginal abatement cost in Chinese regions from 2000 to 2017. Fig. 1 shows the carbon marginal abatement costs in 30 provinces from 2000 to 2017.

It can be found that from 2000 to 2017 , the value of MAC showed an upward trend, and the average value was 1,79.26 yuan per ton, which means that the cost of reducing every 1 ton of carbon emission averagely is $1,79.26$ yuan.

In terms of the regional average MAC at different stages, the eastern regions have the highest average annual MAC from 2000 to 2005, while the western regions have the highest average annual MAC from 2006 to 2011 and 2012-2017. From 2000-2005, the provinces with higher carbon reduction costs are Guangdong, Zhejiang, Jiangsu,
Shandong, and Shanghai. Most provinces' economy is developed. From 2006-2011 and 2012-2017, The provinces with high carbon reduction costs were mainly Qinghai, Hainan, Ningxia.

In terms of the annual average MAC in different regions. The annual average MAC varies significantly among provinces. Shanxi, Hebei, and Shandong have lower average carbon abatement costs, which result from Shanxi has abundant coal resources, and the heavy industry in Shandong and Hebei accounts for a relatively large proportion of the industrial structure. Especially, Shandong's carbon emissions rose to the highest level in the country in 2017. As previous environmental studies founded, the reduction of pollutant emission has a scale effect, which means that the higher the initial emission of pollutants, the lower the marginal emission reduction cost. Provinces such as Qinghai, Hainan, and Beijing have higher average carbon abatement costs, especially, the highest province is Qinghai, which because of the economy of Qinghai and Hainan mainly depend on tourism, and the heavy industry is undeveloped.

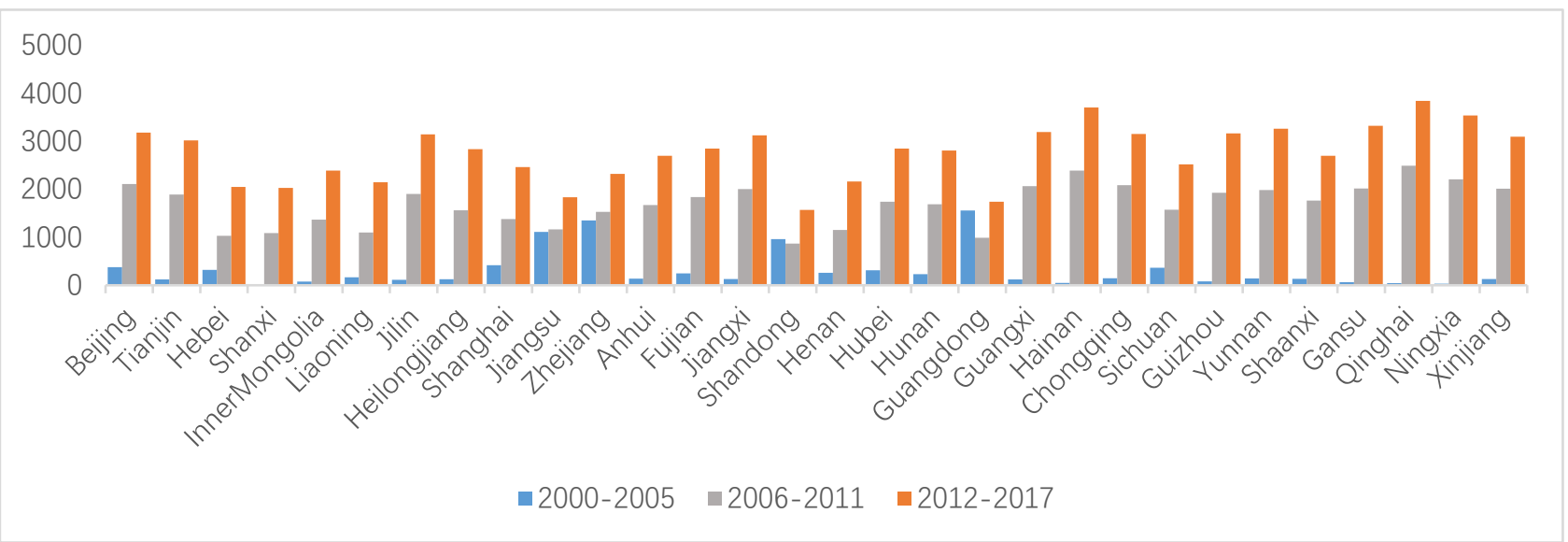

Fig. 1 Distribution of carbon abatement costs

\subsection{Time series characteristics of the carbon abatement cost in three regions}

Considering the differences in geographical location and economic development in various regions in China, this paper divides 30 provinces into three groups (eastern, middle, and western) based on the classification of the China National Bureau of Statistics. The results are shown in Fig. 2.

The curve of MAC kept level in the western, central, and eastern provinces from 2000 to 2006, then all of them rose rapidly from 2006 to 2008. Finally, MAC grows slow in each region after 2008 . The estimated carbon marginal abatement cost in western provinces is higher than that of the other regions, followed by the central region, and the lowest carbon marginal abatement cost is in the eastern region. The carbon marginal abatement costs of western, central, and eastern provinces are 1,726.94 yuan per ton, 1,479.97 yuan per ton, and 1,496.53 yuan per ton, respectively.

The eastern region's economy is more developed, the technology is more advanced, and the total factor productivity is higher. Thus, the carbon abatement cost is lower than that of the other two regions. The western region is an underdevelopment region, and the relative poverty makes more room to reduce carbon emission.

The estimated carbon marginal abatement cost in western provinces is higher than that of the other regions, followed by the central region, and the lowest carbon marginal abatement cost is in the eastern region. The carbon marginal abatement costs of western, central, and eastern provinces are 1,726.94 yuan per ton, 1,479.97 yuan per ton, and 1,496.53 yuan per ton, respectively.

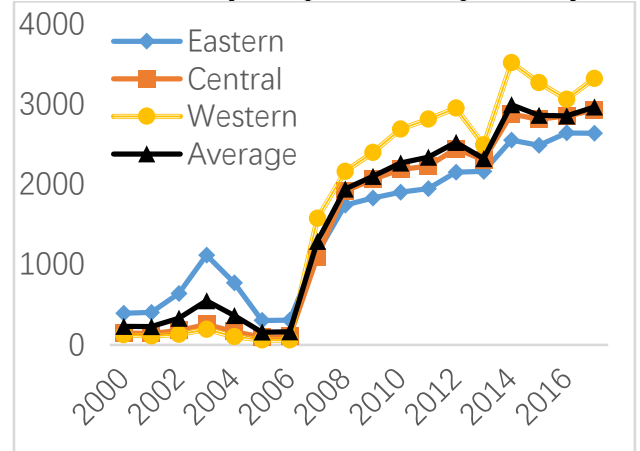

Fig. 2 Carbon abatement costs in different regions 


\subsection{Dynamic evolution characteristics}

Fig. 3 shows the result of Kernel density estimation (KDE) of carbon abatement cost for 2000、2003、2006、2009, and 2012, which indicates the dynamic evolution characteristics of provincial MACs. The result of the dynamic research reflects the variation in the provincial gap of MAC and illustrates the features of MAC. According to Fig. 3, the following features are evident. Firstly, see from the trend of the KDE curve, the curves and centers moved slightly to the right from 2000 to 2017, indicating that the MAC gradually increased from 2000 to 2006, and experienced a significant increase after 2006. Secondly, see from the kurtosis of the KDE curve, the peaks and ranges of different curves experienced varying degrees of change. The modes evolve from a sharp to wide one during the study period, with the height descending, significantly, the dispersion range rapidly widened after 2006, with the height descending rapidly, revealing that the regional gap among MAC of different provinces was enlarging at this stage. Thirdly, see from the shapes of the KDE curve, the curves were Multi-modal before 2006, and with several lumps in the long right tail, while those after 2006 were unimodal, which means that bi-polarization tendency was weakened during the study period.

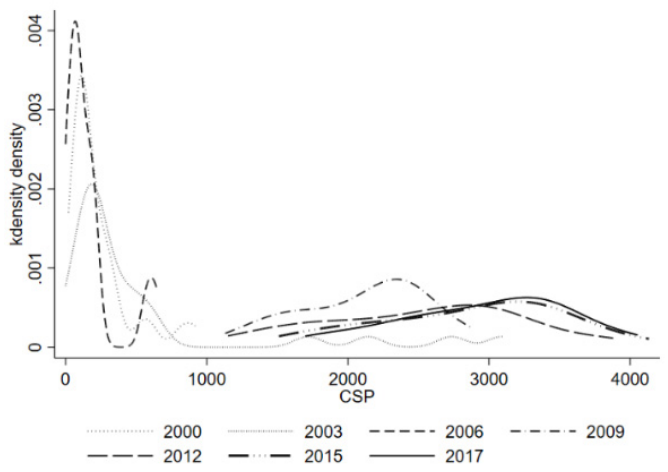

Fig. 3 Dynamic revolution of carbon abatement costs

\subsection{Spatial autocorrelation}

\subsubsection{Global spatial autocorrelation}

Table 1 shows the Moran's I of MAC based on geography spatial weight matrix. Moran's I is not significant before 2008 , while Moran's I is significantly positive at $5 \%$ level after 2008, which indicates that there is a significant positive spatial agglomeration in MAC in China after 2008.

Table 1 Moran's I of carbon abatement costs

\begin{tabular}{cccccc}
\hline Year & Moran's I & P & Year & Moran's I & P \\
\hline 2000 & 0.070 & 0.122 & 2009 & 0.183 & 0.012 \\
2001 & 0.064 & 0.135 & 2010 & 0.194 & 0.009 \\
2002 & 0.046 & 0.176 & 2011 & 0.201 & 0.007
\end{tabular}

\begin{tabular}{llllll}
2003 & 0.033 & 0.220 & 2012 & 0.189 & 0.010 \\
2004 & 0.040 & 0.202 & 2013 & 0.177 & 0.014 \\
2005 & 0.075 & 0.117 & 2014 & 0.200 & 0.007 \\
2006 & 0.075 & 0.116 & 2015 & 0.197 & 0.008 \\
2007 & 0.078 & 0.122 & 2016 & 0.151 & 0.026 \\
2008 & 0.176 & 0.014 & 2017 & 0.184 & 0.011 \\
\hline
\end{tabular}

\subsubsection{Local spatial autocorrelation}

The results of Local indicators of spatial autocorrelation (LISA) agglomeration of MACs showed in Fig.4. Fig.4 shows that there are four types of spatial agglomeration, including $\mathrm{H}-\mathrm{H}$ agglomeration, L-L agglomeration, H-L agglomeration, and L-H agglomeration. From 2008 to 2017 , the MAC of most provinces were auto-correlated, and most provinces locate either in quadrant I or quadrant III. Due to the clustering of provinces with high MAC and low MAC, respectively, the spatial polarization feature appeared. Besides, there are some changes from 2008 to 2017; for instance, Shannxi transformed from $\mathrm{H}-\mathrm{H}$ agglomeration in 2008 to L-H agglomeration in 2017, as might be resulted from developing new sustainable energies to reduce the carbon reduction cost, which means while its neighbors high, the carbon abatement costs of Shannxi is low.

\section{Conclusion}

The rapid economic growth in China caused environmental degradation in the past few decades. The Chinese government is trying to reduce carbon emissions through legislation, administration, and economic tools to develop sustainability. The estimation of the marginal carbon abatement cost can provide valuable information for accurate policymaking.

Using the consumption data of seventeen kinds of fossil fuels from 2000 to 2017, we estimate China's carbon emission more accurately. Taking labor, capital, and energy as input, real GDP as desirable output, and carbon emission as undesirable output, this paper estimates the marginal carbon abatement costs using the directional distance function and analyses the spatial distribution pattern of MAC. Our estimate yields several findings. Firstly, carbon abatement costs in every province of China rise from 2000 to 2017. Secondly, the distribution of MAC shows MAC in western provinces is higher than it in central areas, while a higher MAC in central provinces than in eastern ones. Thirdly, the dynamic evolution characteristics show that MAC increases significantly and the MAC regional gap enlarging after 2006. Finally, the spatial distribution of carbon abatement costs in China shows a geographical clustering feature, and the positive spatial agglomeration is significant after 2008. Local spatial autocorrelation results suggest that the majority agglomeration pattern did not change between 2008 and 2017. 

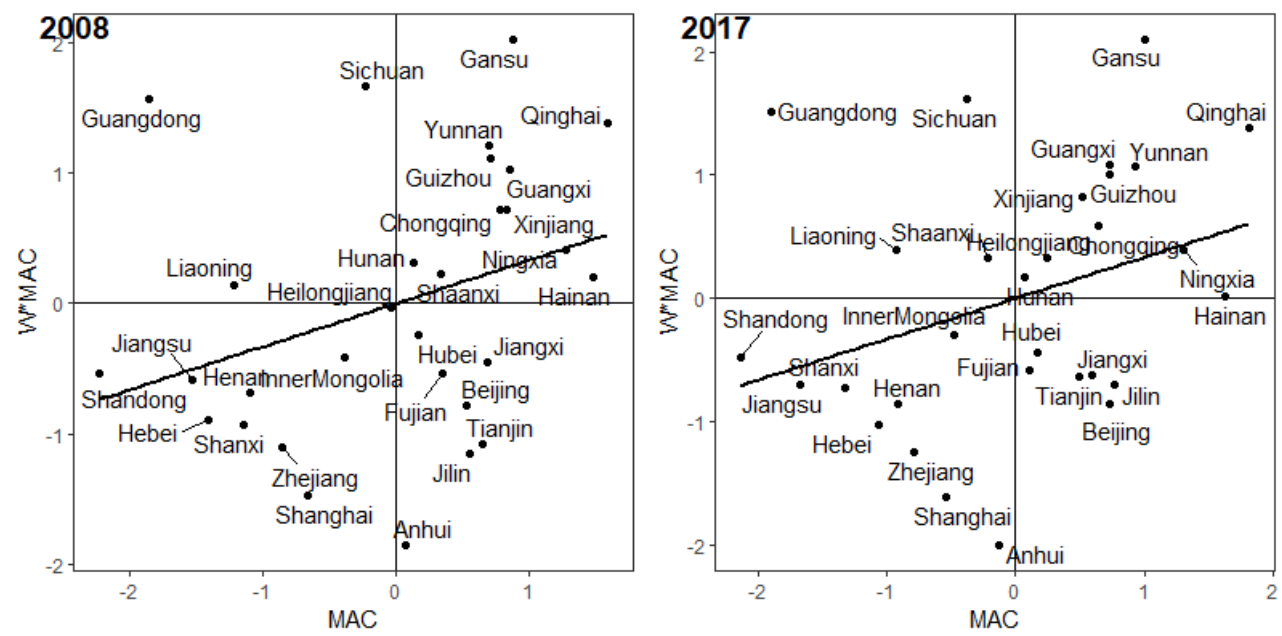

Fig. 4 Moran Scatter plot

\section{References}

1. Y. Choi, N. Zhang, and P. Zhou, Efficiency and abatement costs of energy-related $\mathrm{CO}_{2}$ emissions in China: A slacks-based efficiency measure, Appl. Energy, vol. 98, pp. 198-208, Oct. 2012, doi: 10.1016/j.apenergy.2012.03.024.

2. F. Duan, Y. Wang, Y. Wang, and H. Zhao, Estimation of marginal abatement costs of $\mathrm{CO}_{2}$ in Chinese provinces under 2020 carbon emission rights allocation: 2005-2020, Environ. Sci. Pollut. Res., vol. 25, no. 24, pp. 24445-24468, Aug. 2018, doi:

\subsection{7/s11356-018-2497-x.}

3. Z. Xue, N. Li, H. Mu, M. Zhang, and J. Pang, Convergence analysis of regional marginal abatement cost of carbon dioxide in China based on spatial panel data models, Environ. Sci. Pollut. Res., Mar. 2021, doi: 10.1007/s11356-021-13288-9.

4. R. Färe, S. Grosskopf, C. A. K. Lovell, and S. Yaisawarng, Derivation of Shadow Prices for Undesirable Outputs: A Distance Function Approach, Rev. Econ. Stat., vol. 75, no. 2, pp. 374-380, 1993, doi: $10.2307 / 2109448$.

5. L. Anselin, Local indicators of spatial associationLISA, Geogr. Anal., vol. 27, no. 2, pp. 93-115, 1995. 